\title{
Linx
}

Revue des linguistes de l'université Paris X Nanterre

$62-63 \mid 2010$

Entre syntaxe et discours. Éclairages

épistémologiques et descriptions linguistiques

\section{Retour sur le tour hypothétique qui veïst}

Nathalie Bragantini-Maillard

\section{OpenEdition}

Journals

Édition électronique

URL : http://journals.openedition.org/linx/1384

DOI : 10.4000/linx.1384

ISSN : 2118-9692

Éditeur

Presses universitaires de Paris Nanterre

\section{Édition imprimée}

Date de publication : 1 juillet 2010

Pagination : 181-196

ISSN : 0246-8743

\section{Référence électronique}

Nathalie Bragantini-Maillard, « Retour sur le tour hypothétique qui veïst », Linx [En ligne], 62-63 | 2010,

mis en ligne le 04 décembre 2013, consulté le 20 avril 2019. URL : http://journals.openedition.org/

linx/1384; DOI : 10.4000/linx.1384 


\title{
Retour sur le tour hypothétique qui veïst ${ }^{1}$
}

\author{
Nathalie Bragantini-Maillard \\ Université catholique de Louvain
}

Le tour qui veïst fait partie de ces marques énonciatives dont usèrent les auteurs médiévaux pour impliquer le lecteur-auditeur dans l'action narrée à titre de témoin potentiel. Il partage le rôle avec en particulier es vos et lors veïssiés, sans qu'il y ait toutefois une nécessaire interchangeabilité entre les différentes formules. Chacune conserve en propre ses caractéristiques, notamment syntaxiques, sémantiques et pragmatiques.

S’agissant de qui veïst, plusieurs développements lui ont été consacrés. L'accord paraît unanime sur son sens hypothétique, ainsi que sur son lien, au moins originel, avec le genre épique, de sorte que la critique tend à perpétuer l'appellation de «formule épique » dont Wagner (1939, p. 211)2 baptisa le tour dans son étude sur les phrases hypothétiques commençant par si. En revanche, le statut de ses différentes constructions demeure problématique. Au moins deux questions restent ouvertes :

1- Dans quelle mesure les deux grandes constructions de qui veïst, autonome ou enchâssé dans une principale - ou sur un plan logique, avec ou sans apodose -, sont-elles distinctes? S'il y a divorce, est-il d'ordre syntaxique, sémantique et/ou pragmatique?

\footnotetext{
${ }^{1}$ Il m'est agréable de remercier Pierre Kunstmann, dont le regard d'expert sur la question m’a été très précieux.

2 Précisons toutefois que Kunstmann, 1990, n'emploie pas l'expression de formule épique pour qui veïst, qu'il considère, d'un strict point de vue syntaxique, comme une construction grammaticale.
} 
2- Dans quelle mesure le tour qui veïst prend-il une valeur exclamative? Cette valeur varie-t-elle en fonction du contexte d'emploi, du genre ou d'autres facteurs?

Nous proposons ici nos conclusions à l'issue du sondage d'un corpus de textes composés ou copiés entre le XIe et la fin du XV siècle, sans distinction de genres ${ }^{3}$.

\section{Qui veïst... avec ou sans apodose : équivalence ou spécialisation des constructions?}

Dès les premiers textes, la relative nominale hypothétique qui veïst se rencontre à la fois comme structure autonome et comme constituant d'une principale, constituant à fonctions variables, mais correspondant toujours à la protase d'un système hypothétique complet. Nos prédécesseurs tendent à lier la différence syntaxique entre ces deux emplois à une différence sémantique et pragmatique, réservant l'effet de sens exclamatif à la structure sans apodose 4 . Si l'on admet une "différence de surface» (Kunstmann, 1990, p. 362) - qui nous semble être syntaxique, mais non sémantique, puisque, quelle que soit sa construction, qui veïst ne se départ jamais de son sens hypothétique - et si l'on reconnait à l'emploi autonome une valeur exclamative, quelles sont les spécificités et la valeur de son emploi avec principale ? Chaque construction subit-elle une spécialisation, sans échange possible?

Selon nous, malgré des différences syntaxiques évidentes, le rapport de parenté originel entre les deux constructions est trop prégnant pour que l'on puisse rejeter d'un revers de main une équivalence entre elles. L'analyse des relevés apporte de nouvelles pièces en faveur de cette hypothèse.

\subsection{Structure interne du tour qui veïst}

Les deux constructions du tour qui veïst se livrent une forte concurrence d'un point de vue général avec 109 occurrences en emploi autonome contre 122 en principale. Elles se talonnent tout autant dans le détail de leur structure interne.

Introduit par qui (que dans les textes de l'Ouest), pronom relatif autarcique indéterminé en fonction sujet, le tour a pour noyau le verbe de perception veoir, moins souvent oïr ou reveoir, au subjonctif imparfait (très rarement au subjonctif plus-queparfait). Il est fréquent qu'un anaphorique adverbial (adont, donc, dont, lors...) et/ou pronominal antéposé au verbe ouvre la proposition et assure un enchainement souple avec l'énoncé qui précède 5 . Le verbe de perception est le plus souvent pourvu d'une expansion. Quoique très variables dans le détail, les expansions correspondent à trois grandes constructions : 1) GN COD, seul ou en série énumérative (ex. 1) ; 2) GN COD + Constituant prédicatif [GAdj., GPrép., Inf., Relative] (ex. 2, 5) ; 3) Complétive

\footnotetext{
${ }^{3}$ Voir infra Corpus exploré.

${ }^{4}$ Voir Wagner, 1939, p. 210 ; Moignet, 1959, II, p. 417 ; 1974, p. $167 ; 1985^{3}$, p. 134, note 1680 ; Kunstmann, 1990, p. 362 ; Ménard, 19944, \ 76b, Rem. 1 ; Andrieux-Reix, 1995, p. 136 ; Buridant, 2000, \536, p. 637.

5 Voir Kunstmann, 1990, p. 363.
} 
exclamative, dont le sujet est souvent disloqué par antéposition à la subordonnée et analysable comme premier COD du verbe de perception, par anticipation : "Qui la veïst Rainuart le baron/ com il se fiert entre la gent Mahom $!{ }^{6}$.

S'agissant des tendances partagées, on constate qu'une grande majorité de constructions sont fondées sur l'infinitif (178 occ. sur 231) et que la préférence va à la structure avec adverbe anaphorique (dont, lors, etc.) (143 occ.). Les différences sont, quant à elles, infimes. C'est à peine si l'on décèle une variété combinatoire plus grande quand qui veïst est autonome.

\subsection{Contextes d'emploi}

La différence tient-elle au contexte d'emploi ? L'enquête montre que non. Qui veïst suivi d'une apodose couvre pratiquement les mêmes registres et les mêmes actions qu'en construction autonome.

Nos prédécesseurs ${ }^{7}$ ont insisté sur le lien existant entre qui veïst et la thématique guerrière, la formule, avec ou sans apodose, servant volontiers à animer la narration d'une action guerrière en soulignant le paroxysme d'un combat. La mise en scène d'une action collective est pareillement favorable à l'apparition du tour en tout contexte. Soulignons néanmoins qu'en comparaison avec lors veïssiés, qui veïst est moins souvent associé à la fête. La troisième thématique propice à une présentation en qui veïst est l'expression humaine, c'est-à-dire la manifestation affective (douleur, retrouvailles, rire...) et l'expression corporelle (ivresse, ardente prière...).

En dehors de ces contextes récurrents, il est des séquences ou des thèmes marginaux qui témoignent d'une préférence pour la structure avec apodose. En dépit de la diversité qui caractérise l'ensemble, il semble possible de mettre l'emploi de qui veïst sur le compte de l'intensité émotionnelle de la scène. Ainsi de l'archevêque Thomas Becket représenté seul à table, lorsqu'il se cache en Angleterre pour échapper à la prison : "Qui veïst le saint bumme seeir a sun mangier,/ Que il n'aveit od lui ne clerc ne chevalier $[. .],. /$ De pitié l'en poüst trestut le vis muillier ${ }^{8}$. Ou encore de l'évocation superlative de la beauté ou de la laideur:

1. Ki dunc veïst cel large frunt,

E la greve traitice amunt,

Li beaus oils, les regars si dulz;

Mut le tendreie sage e pruz

Ki l'esgardast en mi le vis,

Ke de s'amur ne fust supris. (Ipomedon, éd. A. Holden, 1979, v. 2241-46)

\footnotetext{
${ }^{6}$ Moniage Rainouart, éd. G.A. Bertin, 1973, t. I, v. 4019-20. C'est nous qui soulignons dans les citations.

${ }^{7}$ Voir Wagner, 1939, p. 211 ; Andrieux-Reix, 1995, p. 136 ; Buridant, 2000, \536.

${ }^{8}$ Guernes de Pont-Sainte-Maxence, La Vie de saint Thomas Becket, éd. E. Walberg, 1936, v. 2071-75.
} 
Enfin, les idées de rapidité et/ou d'effort, sous-jacentes dans les scènes collectives, peuvent devenir centrales, comme dans cette description des éléments déchaînés :

2. Qui lors veïst de toutes pars

Espartir mervilleusement

Et tonner tres horriblement,

Venter, gresler, et fort plouvoir

Les nues, la mer esmouvoir,

Bois trambler, rivieres courir [...],

C'estoit chose trop mervilleuse,

Trop doubtable et trop perilleuse! (Guillaume de Machaut, Jugement dou roy

de Navarre, éd. E. Hoepffner, 1908, t. I, v. 286-296)

\subsection{Valeur pragmatique}

L'examen des contextes d'emploi confirme que le narrateur usant du tour veut attirer l'attention du lecteur-auditeur par une interpellation indirecte, sur un événement ou un objet digne d'intérêt pour son intensité dramatique ou émotionnelle. Il joue la carte de l'affectivité, en exprimant ses impressions. La valeur illocutoire d'un énoncé en qui veist est expressive. En ce sens, qui veïst est toujours susceptible de prendre une valeur exclamative, et ce, quelle que soit sa construction.

Il n'est d'ailleurs pas rare que le tour comporte au moins un élément propre à lui conférer une valeur exclamative (déterminant/adverbe intensif, exclamative indirecte/ directe). Cette valeur peut être confortée par d'autres procédés (morpho)syntaxiques, non porteurs de sens exclamatif en soi, mais opérant comme renforts, tels l'énumération de qualifiants, de verbes d'action ou encore l'emploi du démonstratif de notoriété9. Mais lorsque qui veïst est suivi d'une apodose, comment concilier la valeur exclamative qu'il peut prendre avec son intégration dans une proposition supérieure ? Attardons-nous sur ce qui caractérise l'apodose.

D’une manière générale, celle-ci, souvent modalisée par le semi-auxiliaire pooir, plus rarement devoir, exprime la réaction que serait censée provoquer la perception du procès énoncé par la relative en qui veïst. Plus ou moins explicitement, la réaction implique un jugement ou une appréciation affective, dénotés par un verbe d'évaluation, d'énonciation, d'affect ${ }^{10}$ ou connotés par un verbe de perception, de souvenir, voire plus sporadiquement de réaction physiologique ou d'action. L'allusion à une réaction peut enfin passer par une construction recherchée du type dur cuer eïst se de pitié ne fust meïs, irréel au second degré, qui à la condition initiale (qui veïst...) superpose l'hypothèse d'une réaction contraire à l'effet escompté et ses implications. Par un détour euphémisant, le procédé intensifie l'énoncé et accroit son impact sur l'interlocuteur. Autrement dit, l'apodose vise avant tout à qualifier ce que décrit la protase qui veïst.

\footnotetext{
${ }^{9}$ Voir Buridant, 2000, $\$ 102$.

10 Nous employons affect comme un terme générique incluant les émotions et les sentiments ; sur la difficulté de définir ces trois notions, voir en particulier Novakova et Tutin, 2009; Blumenthal, 2009 ; Flaux et Van de Velde, 2000.
} 
Elle en exprime la valeur morale ou émotionnelle, par l'explicite ou l'implicite. Quoi qu'il en soit, l'énoncé est d'ordre affectif. D'ailleurs, l'apodose comporte la plupart du temps des marqueurs exclamatifs ou à potentiel exclamatif (antéposition de l'adverbe bien ou d'un groupe qualificatif, élément hyperbolique ou superlatif, exclamative directe, etc.). En définitive, la valeur de l'apodose rejoignant la valeur exclamative perceptible dans l'élément initial qui veisst, la phrase, en dépit d'une construction syntaxique de type déclaratif, parait toujours correspondre à une exclamation ${ }^{11}$. Quand elle se clôt sur un discours direct, c'est du reste celui-ci qui prend en charge l'exclamation, matérialisée par une parole vive anonyme : "Qui veïst la entalenté/ Le roy Artu de joie faire/ bien peuist dire en son afaire:/ 'Veci roy plain de toute honnour!" ${ }^{12}$.

Quelle que soit sa construction, le tour qui veïst entre donc dans des énoncés expressifs, qui visent à impliquer émotionnellement le destinataire dans l'action narrée ou l'objet considéré et qui exploitent pour cela l'expression intensive à valeur exclamative. Toutefois, une différence notable distingue les deux constructions : qui veïst en emploi autonome est privé du prédicat contenu dans l'apodose, c'est-à-dire des implications émotives ou morales de ce qu'il désigne. Mais la différence se situe-t-elle vraiment à ce niveau?

Nous avons souligné que l'apodose pose un jugement affectif sur ce que présente la protase qui veïst. Or, les termes de cette appréciation relèvent le plus souvent de la qualification conventionnelle : un héros décrit dans l'acharnement et l'impétuosité du combat est qualifié de vaillant, un personnage en pleurs inspire la pitié, etc. Bref, l'apodose ne fait rien d'autre que formuler l'hyperonyme, le type dont qui veïst développe certains traits contextualisés. Autrement dit, la qualification relève de l'évidence, du lieu commun. Elle est, pour ainsi dire, déjà entendue avant même d'être énoncée. Qualité allant de soi, elle pourrait tout à fait s'exprimer par l'implicite. Or, n'est-ce pas ce qui se passe quand qui veïst est employé de manière autonome? Quand le narrateur d'une scène de combat s'exclame : «Ki veïst... !», n'entend-on pas en sourdine: «on aurait vu un vaillant chevalier!»? Ou plutôt n'espère-t-il pas susciter dans l'auditoire l'émotion qui fera naître cette réflexion si convenue ? En général, on peut dire que l'expression spontanée de l'émotion ne recherche guère l'originalité. Au moins dans un premier temps, le locuteur use de termes stéréotypés

\footnotetext{
11 L'affirmation d'Andrieux-Reix (1995, p. 136), selon laquelle « seule l'absence de cette corrélation [...] autorise l'interprétation exclamative de qui lors veïst», repose, selon toute apparence, sur une approche strictement syntaxique. De ce point de vue, nous ne la récusons pas. Cependant, l'exclamation, qui emprunte sa structure et ses éléments morphologiques à la fois à la phrase déclarative et à la phrase interrogative, n'a pas de réelle spécificité syntaxique. "Manifest[ant] une modalité subjective et exprimant le 'sentiment' du locuteur à l'égard du contenu de son énoncé » (Riegel-Pellat-Rioul, 19995 , p. 390), elle peut s'actualiser en différentes structures et se superposer aux types de phrase. C'est dans cette perspective que nous référons à la notion. Soulignons, au passage, qu'il serait dès lors permis de ponctuer des énoncés construits autour de qui veïst d'un point d'exclamation, pourtant encore trop rarement employé en pareil cas.

12 Melyador, v. 29869-72.
} 
(ex. Formidable!, Quel grand homme !13. Ainsi, quand le prédicat relève du cliché, on complète sans peine la pensée d'un locuteur qui s'écrie Qui veïst...

Le phénomène est très comparable à ce qui se passe en français moderne avec le tour hypothétique $A b !$ si vous aviez vu... Libre au locuteur de faire suivre l'hypothétique d'une appréciation personnelle : Ab! si vous aviez vu ce combat acharné entre les deux boxeurs, vous auriez été admiratif! est réductible à $A$ h! si vous aviez, vu ce combat acharné entre les deux boxeurs!, la réduction pouvant même être extrême : Ab! si vous aviez $v u$ cela! La structure de l'hypothétique tronquée à valeur exclamative existait d'ailleurs déjà en français médiéval ${ }^{14}$ : «Se je les pooie eskiewer/ et mon temps ailleurs alewer $! »^{15}$. Le locuteur compte sur le contexte pour orienter la pensée de l'interlocuteur et laisse deviner ce qui est sous-entendu dans la suspension de l'apodose. Entre eux s'instaure une connivence à la fois topique et émotionnelle.

En somme, la comparaison des deux constructions de qui veïst au plan pragmatique indique qu'il n'y a pas plus de solution de continuité entre elles qu'au niveau de leur structure interne ou de leurs contextes d'emploi. Aussi ne suivons-nous pas Wagner (1939, p. 210, note 2) lorsqu'il distingue entre deux valeurs de la tournure, selon qu'elle apparait dans un texte épique ou dans un texte d'une autre filiation. Pour lui, qui veïst se spécialiserait dans l'expression exclamative de l'affect uniquement dans les textes épiques, en dehors desquels il exprimerait simplement l'irréel du passé (« on aurait pu voir »). Les analyses auxquelles ont été soumis nos relevés ne permettent d'établir aucune distinction de cet ordre.

Si différence il y a, elle se situerait, semble-t-il, dans le degré exclamatif de chaque construction. D'après ce qui s'observe pour $A b$ ! si vous avię vu..., il est possible de supposer une supériorité exclamative dans qui veisst elliptique, par rapport à son emploi avec apodose. Loin de l'anachronisme, l'hypothèse est recevable selon une approche guillaumienne de psychomécanique. Le renforcement de la valeur exclamative de qui veïst autonome procéderait du caractère suspensif de l'énoncé, interrompu au terme de la protase. Le non-dit, en ce qu'il met à contribution la subjectivité et l'imaginaire du lecteur-auditeur, favorise une projection fantasmée, qui intensifie la réception du message et augmente la charge expressive de l'énoncé. En se délestant de sa partie prédicative relevant du lieu commun et en acquérant un statut d'énoncé suspensif et allusif, qui veïst accentuerait sa force exclamative.

L'examen de la valeur exclamative de qui veïst amène à revenir également sur une question soulevée par Andrieux-Reix, qui voit en lors veïssiés une «invitation pressante à un spectacle attendu et grandiose, au récit qui en est fait », tandis que cette valeur «semble moindre avec qui lors veïst» (1995, p. 144). Opinion que nous partageons, à cette nuance près : si lors veïssiés est effectivement une invitation plus

\footnotetext{
${ }^{13}$ Hypothèse plus ou moins intuitive, peu d'études ayant été menées sur le sujet (Bouchard, 2000 ; Manes-Wolfson, 1981 ; Kerbrat-Orecchioni, 1994, t. III ; Traverso, 1993 et 1996). Il faudra toutefois compter à l'avenir sur les résultats qu'obtiendra le groupe de recherches «Typologie de l'expression des émotions ", qui a récemment vu le jour (dir. M.-F. Patte et N. Tersis, Typologie et Universaux Linguistiques, CNRS, 2010).

14 Voir Buridant, 2000, \4 416 et 537 ; Ménard, 19944, \153, Rem. 5.

15 «Si seulement je pouvais éviter [les tourments amoureux] et employer mon temps ailleurs!», Melyador, v. 27242-43.
} 
pressante que qui veïst, cela se justifierait non par une différence de degré, mais par une différence pragmatique. Qui veïst n'invite pas tant à voir et à participer à la narration qu'à partager une émotion, nous l'avons montré. Un autre indice est l'interaction moindre que cette formule indéfinie suppose avec le narrataire, par rapport à lors veïssiés, qui s'adresse directement à la personne de l'interlocution. Qui veïst est pure expression d'une émotion. C'est en ce sens qu'il aurait une valeur exclamative supérieure à lors veïssiés. En clair, chaque formule aurait sa propre fonction pragmatique, que l'autre pourrait approcher sans l'atteindre : lors veïssiés, de valeur illocutoire directive, serait une exhortation pressante à la perception sensorielle (visuelle ou auditive) d'un spectacle et à son récit, tandis que qui veïst, de valeur illocutoire expressive, serait l'expression urgente d'une émotion intense provoquée par un spectacle et son récit. La première formule suppose une implication émotive du narrataire, mais celle-ci reste secondaire ; la seconde formule suppose une perception sensorielle, mais elle n'est qu'un prétexte à l'expression de l'émotion.

\subsection{Distribution générique}

La distribution des deux constructions de qui veïst connaît-elle une spécialisation en fonction du genre ou de l'époque ? Le corpus exclut d'abord l'hypothèse d'une spécialisation générique : jusqu'au XIVe siècle, les principaux genres narratifs (chanson de geste, roman, fabliau, historiographie, histoire religieuse, allégorie, dit) ne marquent pas de préférence perceptible pour l'une ou l'autre structure.

Du point de vue diachronique, dès les premiers textes où la formule est attestée (chansons de geste, romans antiques et de chevalerie), sa construction en phrase autonome côtoie celle avec apodose. L'emploi des deux structures connaît une évolution parallèle, avec une fréquence ascendante aux XII et XIII e siècles, puis une raréfaction, sensible au XIVe siècle, mais qui s'accentue au siècle suivant, avec seulement quatre occurrences dans notre corpus ${ }^{16}$, celles-ci ${ }^{17}$ indiquant que qui veïst ne s'emploierait plus alors qu'avec apodose.

\section{Qui veïst, formule épique ou marque d'oralité ?}

Nous rappelions en introduction l'un des traits définitoires généralement attribués au tour qui veïst, à savoir son rapport au style épique. L'appellation de «formule épique » se justifierait par l'emploi originel du tour «lorsque le récit prend un tour proprement épique », selon Wagner (1939, p. 211). Outre son emploi dans la chanson de geste, son coloris épique lui aurait assuré une place de choix au sein des récits versifiés sous influence épique, tels le roman d'aventures ou les récits de veine réaliste ou historique. Sans nier ce lien avec l'écriture épique, Kunstmann (1990, p. 365) élargit le statut de la tournure qui veïst, qu’il considère comme dédiée à

\footnotetext{
${ }^{16}$ Voir Christine de Pizan, Mutacion de Fortune, éd. S. Solente, 1959, t. I, v. 1967-72 ; Cleriadus, XXVI/162-167 ; Erec en prose, P, V, p. 144 ; Cent Nouvelles nowvelles, éd. Fr. Sweetser, 1966, Nouvelle 83 , p. $487,76-77$.

${ }^{17}$ Les études précédentes n’offrent aucun exemple pour le XVe siècle.
} 
l'animation de la narration dans des textes de style oral et épique. Autrement dit, il souligne le trait d'oralité qu'introduit le tour qui veïst.

Nous irions plus loin. Compte tenu de l'extension générique que le tour connait très tôt, on pourrait se demander si son lien originel avec l'épopée suffit à le définir. Il nous semble que le caractère oral de qui veïst prime sur son caractère épique, qui, pour certains auteurs, a pu ne pas jouer du tout.

\subsection{Extension générique et thématique de la formule}

Kunstmann (1990, p. 364-365), qui s’intéressait à la seule construction elliptique, avait relevé qui veïst uniquement dans le Roman d'Alexandre et dans le Tristan de Béroul, tous deux réputés pour leur coloration épique. Andrieux-Reix (1995, p. 138-139) en avait relevé quelques exemples dans les romans antiques que sont le Brut de Wace et le Roman de Thèbes, invariablement en contexte guerrier. Seuls les romans courtois en vers réorientaient la fonction narrative de la formule en l'employant à l'expression de l'émotion. C'est pourquoi ces premiers relevés confortaient la thèse du caractère épique de qui veïst. Or, nos relevés comblent substantiellement le vide laissé jusqu’à présent dans le genre romanesque : la tournure se rencontre très souvent dans le roman en vers, toutes tendances confondues, et de temps à autre dans la prose romanesque.

Plus précisément, le tour est on ne peut plus compatible avec le roman de veine chevaleresque, arthurienne ou non (Vengeance Raguidel, Durmart le Galois, Cassidorus, Mélusine en prose, Guillaume de Dole, l'Escoufle, Comte d'Artois, etc.). Loin d'apparaitre alors systématiquement en atmosphère épique, il peut servir à dessiner la perfection d'un visage, comme celui de la Fiere dans l'extrait déjà cité d'Ipomedon, ou à souligner la profusion des équipements lors du départ d'Arthur dans le Conte du Graal (éd. F. Lecoy, 1972-1975, v. 4144-49) - vers repris dans Floriant et Florete (v. 3663-67).

$\mathrm{Au}$ sein de la littérature allégorique, qui veïst présente des emplois originaux et ponctue ainsi, lors du banquet du Songe d'Enfer de Raoul de Houdenc, un éloge ironique de la langue des plaideurs, fort appréciée en Enfer pour ses qualités gustatives:

4. Qui veïst com langues aloient

Et ça et la communement,

Mander peüst tout vraiement

Aus parjurez, aus menteors,

Que langues de faus pledeors

Ne sont pas en Enfer blasmees,

Mes chier tenues et amees. (Le Songe d'Enfer, éd. M. T. Mihm, 1984, v. 570-576)

Les textes à vocation religieuse ou hagiographique ne sont pas en reste, puisque le tour parait tout désigné pour y exprimer la ferveur religieuse :

5. Qui lors veïst le menestrel

Le cierge offrir desor l'autel,

Mercier Dieu et Nostre Dame

Dur cuer eüst, foy que doi m'ame, 
Se de pitié ne fust meüz. (Gautier de Coincy, Miracles Notre Dame, éd. V.F. Koenig, 1955-1970, t. IV, Dou cierge qui descendi au jougleour, v. 167-171)

ou la compassion, comme lors de l'assassinat de saint Thomas Becket, où la formule participe à la dramatisation, en introduisant le thème du regard incapable de se détacher du sang et de la cervelle qui, mêlés l'un à l'autre, jonchent le sol. Elle est le point d'ancrage d'un développement qui, sur cinq vers, ressasse l'image obsédante du sang versé (Guernes de Pont-Sainte-Maxence, op. cit., v. 5636-40).

Enfin, la scène théâtrale tire parti des virtualités de la formule. Dans la version dramatique de Griseldis, le marquis, désireux d'éprouver la loyauté de sa femme, exige le sacrifice de son fils. Griseldis se soumet à sa volonté et lui remet l'enfant. Et le marquis de louer l'incommensurable amour qui la caractérise, en dépit du geste cruel qu'elle lui concède: "Mais qui toute jour la veïst,/ Onques maiz ne vi moins amere,/ N'avoir plus grant douceur en mere/ Qu'en lui ont les enfans eü $[\ldots] »^{18}$.

On est donc loin d'une spécialisation dans le genre épique, sans compter que, au-delà de la question générique, on a vu que qui veïst sert à l'expression exclamative de l'affect du narrateur, ce qui, au faite de sa fortune littéraire, le rend compatible, sans connotation archaïsante, avec des sujets esthétiques (description de la beauté féminine), religieux, allégoriques, bref autant de sujets non spécifiques de l'épopée, voire sans rapport avec elle.

\subsection{Une marque d'oralité}

Cela dit, une différence notable se dégage en fonction de la forme textuelle. Qui veïst jouit en effet d'une faveur remarquable auprès des auteurs en vers, tandis que la prose ne lui prête qu'un intérêt plus occasionne ${ }^{19}$. Nos prédécesseurs en ont repéré une occurrence dans les Récits d'un ménestrel de Reims, la Mort le roi Artu et la traduction de Griseldis par Philippe de Mézières, ainsi que cinq dans la chronique de Jean le Bel${ }^{20}$. De notre côté, la moisson est à peine plus riche, avec seulement dix-sept occurrences, dont six fournies par la Mélusine de Jean d'Arras et sept par le Livre I des Chroniques de Froissart ${ }^{21}$, les autres se répartissant entre romans de chevalerie, Cassidorus, Cleriadus et Meliadice et l'Erec en prose, et nouvelle.

Comment expliquer une telle différence de traitement ? Le goût du vers pour la formule qui veïst peut tenir à des considérations métriques, dans la mesure où ce patron syntaxique thématiquement neutre fournit une moitié d'octosyllabe commode à placer en tout contexte. Mais, d'un autre côté, la quasi-indifférence de la prose à son égard

${ }^{18}$ L'Estoire de Griseldis, éd. M. Roques, 1957, v. 1738-43.

${ }^{19}$ Ce constat amènerait à nuancer l'affirmation de Buridant, 2000, \536, p. 637, qui, à la suite de Wagner, continue d'englober les formules épiques du type lors veïssiés, qui veïst pour dire que « le tour perdure dans les romans d'aventure, les récits versifiés ou les mises en prose qui perpétuent les anciennes chansons de geste, ou les chroniques en prose ». Les relevés de Kunstmann, 1990 et les nôtres montrent que, dans la perspective de leur exploitation générique, chaque formule dite épique gagnerait à être prise en compte pour elle-même. Par ailleurs, on ne suivra pas Buridant, 2000, $\ 536$, p. 637, quand il assigne à qui veïst sans apodose le rôle d'exprimer le regret.

${ }^{20}$ Voir Wagner, 1939, p. 214 ; Kunstmann, 1990, p. 364 et 482 ; Andrieux-Reix, 1995, p. 141.

21 Voir Corpus exploré. 
pourrait indiquer que le tour ne répondait pas au souci qui était le sien d'ancrer le récit dans la matérialité de l'écrit, ce qui laisserait penser que l'intérêt que lui prêtait le vers tenait à son caractère oral.

Cela nous ramène à la question du statut épique de qui veïst: si le tour avait été un marqueur typiquement épique, avant d'être un marqueur d'oralité, s'il avait appartenu en propre au répertoire de l'épopée, n'eût-il pas survécu, à titre de reliquat archaïsant, dans la prose épique tardive? Sa disparition peut certes s'expliquer par l'influence qu'exerce le roman sur cette prose, qui verse dans le roman d'aventures. Mais les chansons de geste versifiées prennent également un tour romanesque dès le XIVe siècle, ce qui ne les empêche pas d'user de qui veïst. Ainsi, la formule est encore bien présente dans la version en vers du XVe siècle de la Belle Hélène de Constantinople (voir 2.3). Au contraire, Jean Wauquelin l'évince totalement de sa mise en prose, soit que la formule passe à la trappe avec le développement qui la contient, soit qu'elle est remplacée par une phraséologie tout aussi expressive mais modernisée, à l'instar de ce qui s'observe pour les vers suivants :

6. Qui la veïst le roy tenrement larmïer

Et detordre ses puins et ses cheviaux saquier

Bien peuist dire et croire et pour vrai tesmoignier :

Par foy, chieux nobles rois ama moult se moulier. (La Belle Hélène..., v. 4930-4933)

7. Et, quant il [le roi Henri] eust ce dit, il commença tresfort a plorer et a regreter sa femme et a dire : «Ha! ma treschiere amye [...]» Et veritablement il faisoit de si piteux regrez qu'il n'estoit si dur cuer a le veoir quil ne plorast, tant se lamentoit il piteusement et doloreusement. (Jehan Wauquelin, La Belle Hélène..., LX/58-65)

Son éviction de la prose épique serait un indice de son caractère oral. Déjà au $\mathrm{XIII}$ siècle, le chroniqueur Villehardouin ignore qui veïst, tandis qu'il « glisse dans son récit des veissiez. [...] lorsque le ton de la narration s'élève au niveau de l'épopée », souligne Wagner (1939, p. 212) 22. Serait-ce le signe que lors veïssiés devait être plus déterminant que qui veïst pour le caractère épique d'un texte?

Cependant, toujours dans le genre historiographique, qui veïst a droit de cité chez Jean le Bel, encore que les scènes ne ressortissent pas toujours au registre guerrier $^{23}$. Au siècle suivant, tout en empruntant à son prédécesseur, Froissart tend à se passer de la marque qui veïst en dehors du registre guerrier ${ }^{24}$. Mais lorsqu'il la conserve, il peut renforcer le procédé ${ }^{25}$ et, au fil des réécritures de l'épisode, il revient

\footnotetext{
22 Mais la prose historique délaisse tout autant lors veïssiés dès le XIVe s. ; voir Andrieux-Reix, 1995, p. 140 sqq.

23 Voir en particulier Jean le Bel, t. I, chap. 2, p. 16, chap. 56, p. 318.

24 Voir éd. Luce, I-2, \9, p. 22, 20 ; Manuscrit d'Amiens, I, 15/11 ; Manuscrit de Rome, 5/100 sqq. Pour l'adaptation de Jean le Bel, t. I, chap. 56, p. 318, voir Manuscrit d'Amiens, II, \384 et Manuscrit de Rome, $149 / 71$ sqq.

25 Voir éd. Luce, III, \251, p. 125-126, 31 sqq.; Manuscrit d’Amiens, II, 482/12 sqq.; Manuscrit de Rome, 199/40 sqq.
} 
progressivement à une forme figée du tour. Cette lexicalisation généralisée du tour dans la dernière version du Livre I de ses Chroniques, qui, sous d'autres aspects, se caractérise par une forte stylisation de l'écriture ${ }^{26}$, ainsi que le maintien de qui veïst dans les autres épisodes guerriers laissent penser que le tour fut senti par le chroniqueur comme un élément formulaire stable du répertoire épique. Chez Froissart, le recours à qui veïst aurait donc contribué avant tout à l'atmosphère épique de tel ou tel épisode. Et cela serait vrai aussi bien pour la chronique que pour le roman, puisque les occurrences de Melyador se présentent majoritairement en contexte guerrier.

Pour autant, ce constat ne fait que dégager la sensibilité stylistique d'un écrivain pour une formule donnée, sans préjuger de la signification de celle-ci chez les autres auteurs. En outre, s'il est indéniable que la présence de qui veïst dans les chroniques peut être çà et là un souvenir épique, elle est bien loin de s'imposer dans toutes les scènes traversées par un souffle épique ou de se cantonner dans ce registre. Au fond, elle pourrait tout aussi bien être mise sur le compte du caractère oralisé de la narration - qui est, rappelons-le, l'une des spécificités des chroniques de Jean le Bel et de Jean Froissart. Les observations faites dans ces deux textes n'enlèvent donc rien au fait que la formule ait été opératoire dans des registres aux antipodes de l'épopée, et ce, conjointement aux premières chansons de geste, on l'a vu.

En somme, si les premières attestations de qui veïst sont données par la chanson de geste, ce ne serait pas tant en raison d'une spécialisation épique (animation du récit, valorisation du spectaculaire) qu'en raison d'une appartenance à l'oralité (expression d'un affect, implication du destinataire). Autrement dit, si la chanson de geste exploita la formule peut-être davantage que tout autre genre, ce pourrait bien être avant tout parce qu'elle était originellement destinée à la performance orale. Dès que la prose s'empare de l'épopée, celle-ci tombe dans la matérialité du texte et l'objectivisation du récit et délaisse les effets d'oralité.

D'ailleurs, si rares furent les œuvres en prose à user de qui veïst, encore plus rares durent être celles qui se passèrent de l'apodose. Parmi nos témoins, seuls les Récits d'un ménestrel de Reims $(\$ 123 \text {, p. } 65)^{27}$ et la Mélusine de Jean d'Arras sont dans ce cas. Qu'elle soit romanesque ou historique, la prose tend à maintenir l'apodose, $a$ fortiori quand elle est composée à la fin du Moyen Âge. Cela pourrait tenir au fait que la structure avec apodose, de degré exclamatif moindre, répondait mieux au souci d'objectivisation des faits propre à la prose et au caractère plus impersonnel de l'instance narrative, incarnée par li contes.

De tout ce qui précède on pourrait admettre que le caractère d'oralité de la formule qui veïst préexista à son caractère épique, sans être supplanté par lui quand le chant épique se l'appropria. Certains auteurs, romanciers ou chroniqueurs, purent certes restreindre sa fonctionnalité au champ de bataille. Mais cela fut sans doute une question de choix stylistique personnel, car, à considérer le succès de la formule ailleurs, son emploi en atmosphère épique ne constitua qu'une application stylistique parmi d'autres de cette marque d'oralité.

En raison de sa valeur affective, on pourrait aussi conjecturer que qui veïst est une marque énonciative privilégiée dans les textes à forte présence narratoriale

26 Voir en particulier Bragantini-Maillard, 2012.

${ }^{27}$ Voir aussi Kunstmann, 1990, p. 364 et 482 (Philippe de Mézières, L'Histoire de Griseldis). 
affective, ceux où le narrateur est enclin à rendre compte de ses impressions émotionnelles sur les faits qu'il raconte. Le caractère exceptionnel de la tournure dans un roman en vers comme Escanor, dont l'instance narrative demeure relativement discrète, inciterait à le penser. Ce serait un indice supplémentaire que le choix d'user de la formule ou d'en user avec plus ou moins de générosité relèverait moins d'un code générique que d'un style d'auteur. Mais la question du rapport entre les modalités de présence de la voix narrative d'une part et l'emploi et la fréquence du tour exclamatif qui veïst de l'autre demanderait un examen à elle seule. L'hypothèse reste donc à confirmer.

\subsection{Une expression recherchée?}

Une dernière constante traverse les âges: abstraction faite des nuances pragmatiques qui les distinguent, qui veïst semble vite devenir minoritaire par rapport à lors veïssier, l'écart se creusant au fil des siècles. Ainsi, à la fin du XIVe siècle, dans un roman en vers comme Melyador, qui veisst (chaque fois avec apodose) apparait trois fois moins que le type lors veïssiés ${ }^{28}$. Dans la Belle Hélène de Constantinople en vers, selon les copies, la proportion varie de cinq à six fois moins d'occurrences de qui veïst. Au $\mathrm{XV}^{\mathrm{e}}$ siècle, la prose de Cleriadus et Meliadice accorde une présence dérisoire à qui veïst. Mais plus étonnant, dans sa Mélusine en vers, Coudrette ignore totalement notre formule, alors même qu'il rehausse encore la narration d'actions guerrières de lors veïssiez et que la version initiale en prose de Jean d'Arras ne rechignait guère devant l'emploi de qui veïst, même si, là encore, la tournure restait minoritaire. Mais ce dernier fait pour ainsi dire figure d'exception au sein de la prose, où toute trace de qui veïst tend à être éliminée.

Nous n'interpréterions pas nécessairement la survivance de lors veïssięà qui veïst comme la résolution d'une rivalité ancienne et donc comme le signe d'une interchangeabilité originelle. Outre la nuance pragmatique qui sépare les deux formules (voir 1.3), leur champ d'application thématique ne se recoupe pas toujours. Si toutes deux se partagèrent des thèmes (bataille, fête, émotion), qui veïst étendit son champ d'action à des domaines où il régna sans concurrence attestée de son homologue (abondance de biens, beauté d'un chant, du corps...). Les siècles passant, à aucun moment lors veïssiez. ne parait prendre en charge les emplois qui étaient l'apanage de qui veïst et demeure cantonné dans ses thématiques originelles. Il semblerait même que les textes tardifs réservent à qui veïst l'expression de la douleur, pour laquelle il marquait déjà une certaine inclination dès les premiers textes. Ainsi, dans Cleriadus et Meliadice, l'unique occurrence de qui veïst sert à rendre compte du caractère poignant de la peine qui s'empare de Clériadus à l'annonce de la mort supposée de sa bien-aimée, tandis que la veissiez s'en tient à des scènes de combat, de fête ou de retrouvailles. La situation est comparable chez Jean d'Arras ${ }^{29}$.

Par ailleurs, quand les auteurs veulent exprimer l'intensité d'une émotion, ils recourent à d'autres tours que lors veïssiés, du type Présentatif + Nom d'affect

\footnotetext{
${ }^{28}$ Cinq occurrences de qui veïst, contre quinze pour lors veïssés. Pour ce texte et les autres cités par la suite, le détail du relevé est donné dans le Corpus exploré.

${ }^{29}$ Voir Mélusine, p. 736 et 372.
} 
(«C'estoit grant pitié de veoir departir les troys cousins germains », Cleriadus, XLI/8990). Il est donc difficile d'attribuer la disparition de qui veïst à une concurrence que lui aurait faite lors veïssiés.

Revenons à ce que nous soulignions plus haut, à savoir que les textes tardifs du $\mathrm{XV}^{\mathrm{e}}$ siècle donnent visiblement l'exclusivité à la construction avec apodose. Quelle interprétation donner au phénomène? Une fois que l'expression a vieilli et s'est raréfiée, ses éléments topiques ont dû perdre leur caractère d'évidence. La restitution du prédicat par le destinataire n'allant plus de soi, il devenait nécessaire d'expliciter ce prédicat, donc de rétablir l'apodose, dont on pouvait si facilement se passer autrefois. Serait-ce le signe que le tour n'était plus usité par les locuteurs, qu'il était devenu une formule strictement littéraire, voire un archaïsme littéraire, seul compris par quelques familiers des anciens textes?

A contrario, l'exploitation encore intense de lors veïssiés signalerait-elle que le tour était encore usuel en dehors de l'écriture littéraire? Andrieux-Reix (1995, p. 145) propose de considérer cette marque comme l'indice d'une littérarité. Ce statut expliquerait la faveur que connut la formule auprès du vers en tout genre et de la prose romanesque et, au contraire, sa disparition précoce de la prose historique. Il expliquerait également la stéréotypie thématique de la formule et le figement de sa structure, sur laquelle l'évolution de la langue n'eut pas de prise (Andrieux-Reix, 1995, p. 141 et 143). Mais pour être si souvent employé au moins jusqu’à la fin du XIVe siècle et conserver son efficacité pragmatique, il fallait bien que lors veïssiés eût des chances d'être pleinement compris par le lecteur, ce qui impliquerait qu'il était encore vivant dans l'usage.

L'emploi plus sporadique et la désuétude plus rapide de qui veïst permettraientils d'avancer que ce tour était senti comme plus sophistiqué que son homologue ? C'est fort possible, d'abord parce que, avec apodose, il est d'une structure, en deux temps, plus contraignante que lors veïssiés, simplement introductif. D'autre part, qui veïst a pu être perçu comme moins naturel, dans la mesure où, par sa référence à un universel indéfini, il reste dans l'indéterminé, s'adresse à l'interlocuteur, mais de manière indirecte. On est loin de la franche interpellation que réalisent des expressions porteuses de la deuxième personne, telles que lors veïssiés ou es vos. Avec la complexité de sa syntaxe, sa référence indéterminée et indirecte, qui veïst avait des atouts pour conférer une dignité au discours, mais par là même aussi des traits trop prononcés pour résister à la simplification et à l'uniformisation.

\section{Conclusion}

La différence paraît infime entre la structure hypothétique qui veïst avec apodose et celle qui est tronquée. Dans tous les cas, l'énoncé vise l'appréciation affective avec une valeur exclamative effective, l'ellipse de l'apodose ayant un effet intensif, impliquant davantage le destinataire. D'ailleurs, nous verrions volontiers dans qui veïst l'indice d'une oralité (préexistant à son exploitation épique) et d'une implication émotive bilatérale (narrateur-narrataire). Ce statut expliquerait le dédain de la prose pour un tour dont le vers savait tirer parti de l'essence orale, en particulier dans la perspective de la performance orale. Ce statut expliquerait d'autre part la 
fonctionnalité thématique large de la formule, bienvenue en tout contexte suscitant une émotion forte à partager. Sa fréquence d'apparition dépendrait en somme du degré d'implication du narrateur dans le récit et de sa volonté d'extérioriser son émotion pour entretenir une connivence affective et idéologique avec le narrataire. Preuve en serait le goût dont témoigne la littérature d'édification religieuse (hagiographie, mystère, miracle) pour la formule. Qui veïst serait un subjectivème affectif, au sens où l'entend Kerbrat-Orecchioni $\left(2009^{4}\right.$, p. 79 sqq. et 140). Il serait la marque des textes habités par une présence narratoriale forte et affectivement impliquée. Plus précisément, compte tenu de la relative complexité de sa structure morphosyntaxique et de sa référentialité indirecte, il serait la marque des discours oralisés empreints d'une certaine noblesse, dignité ou gravité.

\section{RÉFÉRENCES BIBLIOGRAPHIQUES}

ANDrieux-Reix, N. (1995), «Lors veïssiez, histoire d'une marque de diction », Linx, 32, p. 133145.

Blumenthal, P. (2009), "Les noms d'émotion: trois systèmes d'ordre», Novakova, I. et Tutin, A. (eds.), Le Lexique des émotions, ELLUG, Grenoble, p. 41-64.

Bouchard, R. (2000), "M'enfin !!! Des 'petits mots' pour les 'petites' émotions ? », Plantin, Chr., Doury, M. et Traverso, V. (éd.), Les Émotions dans les interactions, Presses universitaires de Lyon, Lyon, p. 223-238.

Bragantini-Maillard, N. (2012), "Les réécritures de dialogues dans le Livre I des Chroniques de Jean Froissart », Kullmann, D. (éd.), Actes des journées d'études «Réécritures», Toronto, 26-27 mars 2010, Toronto.

Buridant, Cl. (2000), Grammaire nouvelle de l'ancien français, Sedes, Paris.

FlauX, N. ET VAN DE Velde, D. (dir.) (2000), Les Noms en français : esquisse de classement, Ophrys, Paris.

Heinemann, E. A. (1993), L'Art métrique de la chanson de geste. Essai sur la musicalité du récit, Droz, Genève.

Kerbrat-Orecchioni, C. (20094) [1980], L'Énonciation. De la subjectivité dans le langage, Armand Colin, Paris.

- (1990, 1992, 1994), Les Interactions verbales, Armand Colin, Paris.

Kunstmann, P. (1990), Le Relatif-interrogatif en ancien français, Droz, Genève.

MÉNARD, Ph. (19944) [1973], Syntaxe de l'ancien français, Éditions Bière, Bordeaux.

Manes, J. ET Wolfson, N. (1981), «The Compliment Formula», Coulmas, F. (éd.), Conversational Routine, Mouton, La Haye-Paris-New York, p. 115-132.

Moignet, G. (1959), Essai sur le mode subjonctif en latin postclassique et en ancien français, Presses Universitaires de France-Publications de la Faculté des Lettres et des Sciences Humaines d'Alger, Paris-Alger. 
- (1974), Études de psycho-systématique française, Klincksieck, Paris.

- (éd.) (19853) [1972], La Chanson de Roland, Bordas, Paris.

Novakova, I. ET Tutin, A. (eds.) (2009), Le Lexique des émotions, ELLUG, Grenoble.

Perret, M. (1988), Le Signe et la mention. Adverbes embrayeurs ci, ça, la, iluec en moyen français (XIV $V^{e}-X V^{e}$ siècles), Droz, Genève.

Riegel, M., Pellat, J.-Ch. ET Rioul, R. (19995) [1994], Grammaire méthodique du français, PUF, Paris.

Traverso, V. (1993), «Les routines : lieux communs de la conversation », Plantin, Chr. (éd.), Lieux communs, topoi, stéréotypes, clichés, Kimé, Paris, p. 111-122.

- (1996), La Conversation familière, Presses universitaires de Lyon, Lyon.

WAGNER, R.-L. (1939), Les Phrases hypothétiques commençant par si dans la langue française, des origines à la fin du XVTe siècle, Droz, Paris.

\section{Corpus exploré}

Le corpus se compose des textes des bases consultées ${ }^{30}$, auxquels s'ajoutent les suivants ${ }^{31}$ :

Belle Hélène de Constantinople (La), éd. Cl. Roussel, 1995, Droz, Genève [v. 4930-33, 10099-100, 13073-74, 13273-79].

Claris et Laris, éd. C. Pierreville, 2008, Champion, Paris [v. 4918-4921, 4962-4963, 6390-6392, 7081-7084, 7562-65, 8033-40, 15614-16, 16702-04, 30024-27].

Cleriadus et Meliadice, éd. G. Zink, 1984, Droz, Paris-Genève [qui veïst: XXVI/166-167 ; lors veissiez: III/157-158, X/70-73, XXXIV/303-306, etc.].

Coudrette, Le Roman de Mélusine ou histoire de Lusignan, éd. E. Roach, 1982, Klincksieck, Paris [lors veïssiez: v. 1517, 1527, 1563, 2259].

Floriant et Florete, éd. A. Combes et R. Trachsler, 2003, Champion, Champion Classiques, Paris [v. 302-303, 3663-67, 3870-72].

Girart d'Amiens, Escanor, éd. R. Trachsler, 1994, Droz, Genève [v. 13246-52, 13310-13]

Histoire d'Erec en prose (L'), éd. M. Colombo Timelli, 2000, Droz, Genève [qui veïst: P, V, p. 144 ; si össiés: $P$, I, p. 104 ; si eussiéz veu : $B, \mathrm{XXXIX,} \mathrm{p.} \mathrm{206].}$

\footnotetext{
${ }^{30}$ Base de Français Médiéval, 2005, Lyon, UMR ICAR/ENS-LSH. http://bfm.ens-lsh.fr; Base du Dictionnaire du Moyen Français, version 2010, ATILF-CNRS/Nancy Université. http://www.atilf.fr/dmf; Kunstmann, P. et Olsen, M., 2003-2006, Textes de français ancien, Université d'Ottawa-Université de Chicago. http://www.lib.uchicago.edu/efts/ARTFL/projects/TLA ; Kunstmann, P., 2007-2011, Dictionnaire Électronique de Chrétien de Troyes, Université d'Ottawa-ATILF/Nancy Université. http://www.atilf.fr/dect; Stein, A. et al., 2011, Nowveau Corpus d'Amsterdam [A. Dees, 1987], Universität Stuttgart, Institut für Linguistik/Romanistik.

${ }^{31}$ Nous nous bornons à recenser entre crochets les occurrences de qui veïst qui se trouvent dans les témoins consultés en dehors des bases textuelles.
} 
Nathalie Bragantini-Maillard

Jean Bodel, La Chanson des Saisnes, éd. A. Brasseur, 1989, Droz, Genève [v. 216-217AR, 254AR, 271-273AR, 865AR, 2196AR, 3590-3591AR, 4858-4861LT, 286-287R, 457-461R, $945-$ 947R, 976-978R, 1092-1094R, 1451-1455R, 1712-1714R, 1788-1789R].

Jean d'Arras, Mélusine ou la noble histoire de Lusignan, éd. J.-J. Vincensini, 2003, Librairie Générale Française, Paris [qui veïst: p. 314, 360, 422, 696, 736, 742 ; lors veissiez: p. 248, 304, 344, $374,420,476$, etc.].

Jean le Bel, Chronique, éd. J. Viard et E. Déprez, 1904, Société de l'Histoire de France, Paris.

Jean Froissart, Chroniques, Livre I : Le manuscrit d'Amiens, éd. G. T. Diller, 1991, Droz, Genève [II, 362/28 sqq. (2 occ.)] ; Édition du manuscrit de Rome Reg. lat. 869, éd. G. T. Diller, 1972, Droz, Paris-Genève [199/40 sqq. (4 occ.)] ; éd. S. Luce, 1869, Société de l'Histoire de France, Paris [II, \169, p. 153 (1occ.)].

- L'Espinette amoureuse, éd. A. Fourrier, 19631/200233, Klincksieck, Paris.

- Melyador, éd. N. Bragantini-Maillard, 2012, Droz, Genève [qui veïst : v. 1261-64, 18385-87, 16391-96, 29225-29, 29869-72 ; lors veïssiés : v. 1143, 2640, 2641, 3731, 6648, etc.].

Jehan Wauquelin, La Belle Hélène de Constantinople, éd. M.-Cl. de Crécy, 2002, Droz, Genève.

Partonopeus de Blois: An Electronic Edition, éd. P. Eley et al., 2005, HriOnline, Sheffield, www.hrionline.ac.uk/partonopeu [v. 8792-99A, 12065-69G]. 